\title{
Duality of Coordinates and Matter Fields in Curved Spacetime
}

\author{
M. A. De Andrade * \\ Grupo de Física Teórica, Universidade Catolica de Petrópolis (UCP) \\ Rua Barão do Amazonas 124, 25.685-000 Petrópolis-RJ, Brazil \\ I. V. Vancea ${ }^{\dagger}$ \\ Instituto de Física Teórica, Universidade Estadual Paulista (UNESP) \\ Rua Pamplona 145, 01405-900 São Paulo-SP \\ and \\ Departamento de Física Teórica, Universidade do Estado do Rio de Janeiro (UERJ) \\ Rua São Francisco Xavier 524, Maracanã, Rio de Janeiro-RJ, Brazil \\ 02.40.-k, 11.10.+t, 03.70.+k
}

Typeset using REVTEX

*e-mail: marco@cbpf.br

†e-mail: ivancea@ift.unesp.br 


\begin{abstract}
We show that there exists a duality between the local coordinates and the solutions of the Klein-Gordon equation in curved spacetime in the same sense as in the Minkowski spacetime. However, the duality in curved spacetime does not have the same generality as in flat spacetime and it holds only if the system satisfies certain constraints. We derive these constraints and the basic equations of duality and discuss the implications in the quantum theory.
\end{abstract}

According to [1], there exists a duality between the Cartesian coordinates of flat spacetime and the solutions of the equation of motion of a certain physical systems. In the nonrelativistic case, this duality allows to express the coordinates as functionals on the wave functions of an one particle quantum system and some other functionals called prepotentials introduced in [2] (see also [3, (1.) In Minkowski spacetime all the Cartesian coordinates are functionals on the solutions of the classical Klein-Gordon equation and prepotentials.

The results from [1] suggest that the spacetime measurements can be represented in terms of either coordinates or scalar fields, maybe even quantum fields. This interpretation might be particularly useful at the Planck scale where the classical rulers and clocks can be at most idealized while the fields are physical. Also, the Heisenberg inequalities introduce a strong indeterminancy in the spacetime measurements performed by the inertial observers [0] 6]. In particular, this might be a hint that the microscopic structure of spacetime is actually operatorial and noncommutative [7:8]. However, at distances of the order of Planck length, the effects of gravity are rather strong. According to the principles of general relativity, in the presence of gravity the coordinates cease to have the same important role as in the flat spacetime physics [9]. Nevertheless, they are still essential to perform local physical measurements. Therefore, it is important to understand whether the coordinate-field duality described in [1] has a counterpart in curved spacetime.

The purpose of this letter is to investigate the circumstances which allow a duality 
between the local coordinates and the solutions of the Klein-Gordon equation in curved spacetime in both classical and quantum theories (for recent attempts to formulate the gravity in terms of quantum quantities see [10]- [14].) The sought for duality relations should have the same interpretation as in the flat spacetime and should include it as a particular case.

Let us consider a curved spacetime manifold $M$ of dimension $n$, endowed with a Lorentzian metric tensor field $g$ and a scalar field $\phi$ of mass $m$. In a local coordinate system $\left(x^{0}, x^{1}, \ldots, x^{n-1}\right)$ the equation of motion of $\phi$ is given by

$$
\left(\square_{x}+m^{2}+\xi R(x)\right) \phi(x)=0,
$$

where $\square_{x}$ is the d'Alambertian, $\xi$ is a real parameter and $R(x)$ is the scalar curvature of $M$ at $x$. In order to find a duality of the same type as in [1] one has to express the coordinate along a direction $x^{\mu}$ as a function of some solution of (1) for which $x^{\nu}$ (with $\nu \neq \mu$ ) represent independent parameters. These "one dimensional" solutions do not exist in general unless some constraints are imposed on the system. In order to find them we put (11) under the form

$$
M^{(\mu \mu)} \partial_{\mu} \partial_{\mu} \phi\left(x^{\mu}\right)+N^{(\mu)} \partial_{\mu} \phi\left(x^{\mu}\right)+P^{(\mu)} \phi\left(x^{\mu}\right)+Q^{(\mu)}=0
$$

for $\mu=0,1, \ldots, n-1$. The coefficients $M^{(\mu \mu)}, N^{(\mu)}, P^{(\mu)}$ and $Q^{(\mu)}$ are specific functions on $g, \xi$ and $R$, but it is convenients to treat (2) in its full generality and to imposed the dependence later as a constraint. Since the variable in (2) is $x^{\mu}$ while $x^{\nu}$ are treated as parameters for $\nu \neq \mu$ and since we are seeking for independent dualities along each $\mu$, we have to make a first technical assumption that $\phi$ and $\partial_{\nu} \phi$ can be treated as independent functions. As a consequence, the coefficients in (2) can be considered as continuous functions on $x^{\mu}$ depending on the parameteres $x^{\nu}$. The second nontrivial requirement that should be satisfied by the system is that (2) admits two linearly independent solutions. This condition can be translated into a statement about a second order differential equation obtained by changing the coordinates in (2) to local Cartesian coordinates in $R^{n}$ [16]. A general solution 
of the later can be constructed out of a particular integral of (2) and a complementary function that is a solution of the corresponding homogeneous equation. For the later a fundamental solution always exist [15].

The two conditions above refer to the possibility of decomposing (2) along the directions $x^{\mu}$ as in the flat case. In general, it is known that such of decomposition is not possible due to the presence of the off-diagonal elements of the metric in the d'Alambertian. Moreover, even the notion of direction is of little use since there is no invariance of the metric along any vector field [9]. These facts question the existence of any solution to the conditions stated above. However, a large class of manifolds should meet these requirments, namely the manifolds that present at least a number of local Killing vectors equal to the dimension of the manifold. In this case it is known that the Klein-Gordon equation admits wave-function solutions along the integral lines of the Killing vectors [9]. Then the fundamental theorem for linear differential equations [15] guarantees that the homogeneous one-variable equation (2) has two linearly independent solutions and that the inhomogenous equation has solution over the interval where the coeficients $M^{(\mu \mu)}, N^{(\mu)}, P^{(\mu)}$ and $Q^{(\mu)}$ are continuous and defined and $M^{(\mu \mu)} \neq 0$. The existence of the second linearly independent solution depends on the specific form of $Q^{(\mu)}$ which at its turn depends on the particular metric. The fact that $\phi$ and $\partial_{\nu} \phi$ are treated as independent functions implies that any duality relation that can be derived under this assumption is strictly local. Otherwise, a relationship between the two functions can be shown to arise.

Let us assume that the above requirements are satisfied for our system. We denote by $\phi^{\mu}$ and $\tilde{\phi}^{\mu}$ two linearly independent solutions of (2). Following [1] we introduce the prepotentials $\mathcal{F}^{(\mu)}\left[\phi^{\mu}\right]$ by

$$
\tilde{\phi}^{(\mu)}=\frac{\partial \mathcal{F}^{(\mu)}\left[\phi^{\mu}\right]}{\partial \phi^{(\mu)}}
$$

The variation of $\mathcal{F}^{(\mu)}$ with respect to $x^{\mu}$ is given by

$$
\partial_{\mu} \mathcal{F}^{(\mu)}=\frac{1}{2} \partial_{\mu}\left(\tilde{\phi}^{(\mu)} \phi^{(\mu)}\right)+\frac{1}{2} W^{(\mu)},
$$


where

$$
W^{(\mu)}=\tilde{\phi}^{(\mu)} \partial_{\mu} \phi^{(\mu)}-\partial_{\mu} \tilde{\phi}^{(\mu)} \phi^{(\mu)}
$$

is the Wronskian of $\phi^{(\mu)}$ and $\tilde{\phi}^{(\mu)}$. In general, $W^{(\mu)}$ is a continuous functions on $x^{\mu}$ and depends on the parameters $x^{\nu}$ with $\nu \neq \mu$. However, since we want to express $x^{\mu}$ as an explicit function on $\phi^{(\mu)}$ and $\tilde{\phi}^{(\mu)}$ from (4) $W^{(\mu)}$ should be a (nonvanishing) constant function with respect to $x^{\mu}$. This imposes the following supplementary constraint on the system

$$
N^{(\mu)} W^{(\mu)}+M^{(\mu \mu)} Q^{(\mu)}\left(\tilde{\phi}^{(\mu)}-\phi^{(\mu)}\right)=0
$$

for $\mu=0,1, \ldots, n-1$. Now integrating (4) with respect to $x^{\mu}$ we obtain the following relation

$$
x^{\mu}=\frac{2}{W^{(\mu)}}\left(\mathcal{F}^{(\mu)}-\frac{1}{2} \tilde{\phi}^{(\mu)} \phi^{(\mu)}-C^{(\mu)}\right)
$$

for $\mu=0,1, \ldots, n-1$. Here, $C^{(\mu)}$ is an integration constant with respect to $x^{\mu}$ and an arbitrary function on the parameters. Equation (7) expresses the duality between the coordinate $x^{\mu}$ on one hand, and $\phi^{(\mu)}, \tilde{\phi}^{(\mu)}$ and $\mathcal{F}^{(\mu)}$ on the other hand. We note that it has the same form as in flat spacetime, due to the imposed requirements. By setting $W^{(\mu)}$ and $C^{(\mu)}$ constants with respect to $x^{\nu}$ as in the flat spacetime and by using (3) one can see that $x^{\mu}$ depends explicitely only on $\phi^{(\mu)}$ and $\mathcal{F}^{(\mu)}$ which is unknown.

In order to derive the equation satisfied by $\mathcal{F}^{(\mu)}$ we introduce the functional $x^{\mu} \equiv$ $\mathcal{G}^{(\mu)}\left[\phi^{(\mu)}\right]$. Follwing [⿴囗十 we express the derivatives with respect to $x^{\mu}$ in terms of the derivatives with respect to $\phi^{(\mu)}$. We see that

$$
\frac{\partial}{\partial x^{\mu}}=\left(\partial \mathcal{G}^{(\mu)}\right)^{-1} \frac{\partial}{\partial \phi^{(\mu)}}
$$

and

$$
\frac{\partial^{2}}{\partial x^{\mu} \partial x^{\mu}}=\left(\partial \mathcal{G}^{(\mu)}\right)^{-1}\left[-\left(\partial \mathcal{G}^{(\mu)}\right)^{-2}\left(\partial^{2} \mathcal{G}^{(\mu)}\right) \frac{\partial}{\partial \phi^{(\mu)}}+\left(\partial \mathcal{G}^{(\mu)}\right)^{-1} \frac{\partial^{2}}{\partial \phi^{(\mu)} \partial \phi^{(\mu)}}\right]
$$


where $\partial=\partial / \partial \phi^{(\mu)}$. Using (7) we calculate the first two derivatives of $\mathcal{G}^{(\mu)}$ and find the following relations

$$
\partial \mathcal{G}^{(\mu)}=\frac{1}{W^{(\mu)}}\left(\partial \mathcal{F}^{(\mu)}-\partial^{2} \mathcal{F}^{(\mu)} \phi^{(\mu)}\right) \quad, \quad \partial^{2} \mathcal{G}^{(\mu)}=-\frac{1}{W^{(\mu)}} \partial^{3} \mathcal{F}^{(\mu)} \phi^{(\mu)}
$$

Next, by using (3), (3), (9) and (10) in (2) we obtain after some simple computations the following equation

$$
\begin{aligned}
M^{(\mu \mu)} \partial^{3} \mathcal{F}^{(\mu)} \partial \mathcal{F}^{(\mu)} & +\frac{1}{W^{(\mu)}} N^{(\mu)}\left(\partial \mathcal{F}^{(\mu)}-\partial^{2} \mathcal{F}^{(\mu)} \phi^{(\mu)}\right)^{2} \partial^{2} \mathcal{F}^{(\mu)} \\
& +\frac{1}{W^{(\mu)}}\left(P^{(\mu)} \partial \mathcal{F}^{(\mu)}+Q^{(\mu)}\right)\left(\partial \mathcal{F}^{(\mu)}-\partial^{2} \mathcal{F}^{(\mu)} \phi^{(\mu)}\right)^{3}=0
\end{aligned}
$$

for $\mu=0,1, \ldots, n-1$. Equation (11) is the "equation of motion" of $\mathcal{F}^{(\mu)}$ in the space of solutions of (2). Together with (7) it represents the generalization of the duality relations obtained in [1] to a curved manifold. However, (7) and (11) do not have the same generality as in the Minkowski space since we have assumed that the system satisfies three strong mathematical requirements. Actually, in order to make contact with physics, the general coefficients of (2) are connected to the geometrical objects defined on $M$ through the following relations

$$
\begin{aligned}
M^{(\mu \mu)} & =g^{\mu \mu} \sqrt{g}, N^{(\mu)}=\partial_{\mu}\left(g^{\mu \mu} \sqrt{g}\right)+\sum_{\nu \neq \mu} \partial_{\nu}\left(g^{\nu \mu} \sqrt{g}\right) \\
P^{(\mu)} & =\sqrt{g}\left(m^{2}+\xi R\right), Q^{(\mu)}=2 \sum_{\nu \neq \mu}\left(g^{\mu \nu} \sqrt{g} \partial_{\mu} f_{\nu}+\frac{1}{2} \partial_{\mu}\left(g^{\mu \nu} \sqrt{g}\right) f_{\nu}\right) \\
f_{\nu} & =\partial_{\nu} \phi
\end{aligned}
$$

for $\mu=0,1, \ldots, n-1$. Thus we see that (6) represents a constraint on the geometry as well as on the derivatives of $\phi$ with respect to the parameters $x^{\nu}$ with $\nu \neq \mu$.

Our technical assumptions on the non-homogeneous second order differential equation (2) are quite restrictive. Nevertheless, they include the homogeneous equation $Q^{(\mu)}=0$. The relations describing the duality can be directly obtained from the general case. Indeed, from (12) we see that the homogeneous case is obtained for the metrics on $M$ that satisfy the following relation 


$$
\sum_{\nu \neq \mu}\left(g^{\mu \nu} \sqrt{g} \partial_{\mu} \partial_{\nu}+\frac{1}{2}\left(\partial_{\mu}\left(g^{\mu \nu} \sqrt{g}\right) \partial_{\nu}\right) \phi=0,\right.
$$

which represent just the homogenity condition. Also, the condition (6) of having a constant Wronskian becomes in this case

$$
\sum_{\nu=0}^{n-1} \partial_{\nu}\left(g^{\nu \mu} \sqrt{g}\right)=0
$$

which can be obtained from (6) and (12) with the homogenity condition (13). The equation (14) fixes the metric on $M$. When used together with (13) in the Klein-Gordon equation it leads to the following equation for $\phi$ along $x^{\mu}$

$$
\frac{1}{2} \partial_{\mu}\left(g^{\mu \mu} \sqrt{g}\right) \partial_{\mu} \phi+g^{\mu \mu} \sqrt{g} \partial_{\mu} \partial_{\mu} \phi+\left(m^{2}+\xi R(x)\right) \phi=0 .
$$

Equation (15) is a homogeneous differential equation of rank two and it admits always two linearly independent solutions if the coefficients are continuous and nonsingular. The duality relation () has the same form in the homogeneous case, but the equation satisfied by $\mathcal{F}^{(\mu)}$ takes the following form

$$
M^{(\mu \mu)} \partial^{3} \mathcal{F}^{(\mu)}+\frac{1}{W^{(\mu)}} P^{(\mu)}\left(\partial \mathcal{F}^{(\mu)}-\partial^{3} \mathcal{F}^{(\mu)} \phi^{(\mu)}\right)^{3}=0 .
$$

In particular, the Minkowski case studied in [1] can be obtained from the general nonhomogeneous theory by setting

$$
V^{(\mu)}=\frac{1}{\tilde{\phi}^{(\mu)}} Q^{(\mu)}
$$

and by choosing appropriate normalization conditions for $\phi^{(\mu)}$. The corresponding duality relations can be easily deduced from (7) and (11).

Let us discuss briefly the coordinate-field duality in the quantum theory (for more details see [16]). In the operatorial formalism, the general solution of (1) can be expanded as

$$
\phi(x)=\sum_{\alpha}\left(a_{\alpha} f_{\alpha}(x)+a_{\alpha}^{\dagger} \bar{f}_{\alpha}(x)\right)
$$

where $\left\{f_{\alpha}(x)\right\}$ is a local orthonormal and complete set of solutions of (i1) and $a_{\alpha}$ and $a_{\alpha}^{\dagger}$ are the (covariant) annihilation and creation operators, respectively. Since the Poincaré 
group is not a symmetry group of the system, the particle interpretation is problematic and the theory suffers from the known inconsistencies [9]. Nevertheless, let us examine the circumstances under which this theory can display a coordinate-field duality of the same kind as the one discussed in [1]. As in the classical case, in general there is no such of duality in the system. However, one can see that it exists if the Klein-Gordon field admits a local decomposition in independent modes along each of the directions $\mu=0,1, \ldots, n-1$. In particular, this implies that the equation (2) has an orthonormal and complete set of solutions $\left\{f_{\alpha}^{(\mu)}\right\}$, where $\alpha$ represent all the necessary indices to label an independent mode. This linearizing condition strongly constraints the possible types of coefficients to be used in the quantum equation (2) and consequently it constraints the spacetime geometries.

Let us assume that a $\left\{f_{\alpha}^{(\mu)}\right\}$ exists such that it satisfies the following conditions

$$
\begin{aligned}
& f_{\alpha}^{(\mu)} \cdot f_{\beta}^{(\nu)}=-\bar{f}_{\alpha}^{(\mu)} \cdot \bar{f}_{\beta}^{(\nu)}=\delta^{\mu \nu} \delta_{\alpha \beta} \\
& f_{\alpha}^{(\mu)} \cdot \bar{f}_{\alpha}^{(\mu)}=0
\end{aligned}
$$

where "." is the scalar product in the space of solutions defined by an integral over a spacelike hypersuface, then we can construct two linearly independent solutions of (2) of the following form

$$
\phi_{\alpha}^{(\mu)}(x)=a_{\alpha} f_{\alpha}^{(\mu)} \quad, \quad \tilde{\phi}_{\alpha}^{(\mu)}(x)=a_{\alpha}^{\dagger} \bar{f}_{\alpha}^{(\mu)},
$$

which correspond to operators that locally annihilate and create, respectively, the quantum mode $\alpha$ of the field along the direction $\mu$. Using the same definitions (3) as in the classical case, one can associate to each mode $\alpha$ the quantum prepotential $\mathcal{F}_{\alpha}^{(\mu)}$ which is an operator depending on $\phi_{\alpha}^{(\mu)}$.

The existence of a basis (19) restricts the possible manifolds that can support a quantum duality of the type presented in [1]. In particular, let us suppose that $M$ and its geometric structure satisfies all the requirements necessary for a classical duality between coordinates and fields to hold. As we argued previously, depending on $Q^{(\mu)}$, this can take place if there are local Killing vector fields that define the local directions arround $\left(x^{\mu}\right)$ on $M$. In this 
case, the modes $f_{\alpha}^{(\mu)}$ can be taken as the eigenvalues of the translation operator along the Killing vector that defines the direction $x^{\mu}$. In particular this means that $\partial_{\mu} f_{\alpha}^{(\mu)}=k_{\mu} f_{\alpha}^{(\mu)}$ and that the equation (2) is also satisfied by the elements of the basis (for certain functions $Q^{(\mu)}$ the solutions of the inhomogeneous differential equation (2) can be decomposed in the modes of the translation operator, the trivial case being of the homogeneous equations.) Now if we introduce a solution of the form (20) in (2) and expand the function $Q^{(\mu)}$ in the basis (19) with the coefficients $q_{\alpha}^{\mu}$ then we obtain the following equation

$$
q_{\alpha}^{\mu}=-a_{\alpha}\left(k_{\mu} k_{\mu} M^{(\mu \mu)}+k_{\mu} N^{(\mu)}+P^{(\mu)}\right) .
$$

Thus, a basis that satisfies (19) exists on $M$ if the local directions are defined in terms of local Killing vectors and if the classical duality coordinate-fields holds. Also, it is necessary that the coefficients of (2) that depend on the geometry of $M$ satisfy (21) beside (6). The resolution of the basis depends on $Q^{(\mu)}$. The equation (21) admits in general solutions, but these cannot be uniquely determined only from it. However, beside this equation, the functions $M^{(\mu \mu)}, N^{(\mu)}, P^{(\mu)}$ and $Q^{(\mu)}$ should also satisfy (6) but even in this case the solutions are not completely determined even if their existence can be inferred from a simple analysis of the rank of the system. It is possible that a basis of the form (19) exists in a more general or different case, but this problem is not clear to us at present. The basis could be in principle continuous or discrete (for example in the case of a compact Killing vector, the momentum eigenvalues are quantized.)

It is easy to verify that the rest of conditions that should be imposed on the system actually refer only to the modes $f_{\alpha}^{(\mu)}$ and they reduce to the ones discussed in the case of classical fields. This is a consequence of the fact that the modes are assumed to be independent. In particualar, the coefficients $M_{\alpha}^{(\mu \mu)}, N_{\alpha}^{(\mu)}$ and $Q_{\alpha}^{(\mu)}$ that enter the corresponding equation of the form (2) written for the mode $\alpha$ along the direction $\mu$ should satisfy the constraint (6) with $W_{\alpha}^{(\mu)}$ the Wronskian of $f_{\alpha}^{(\mu)}$ and $\bar{f}_{\alpha}^{(\mu)}$. Proceeding along the same line as in the classical case, it turns out that the duality between coordinates and fields is expressed by the following relation 


$$
\begin{aligned}
& X_{\alpha}^{(\mu)}=\frac{2}{W_{\alpha}^{(\mu)}}\left(\mathcal{F}_{\alpha}^{(\mu)}-\frac{1}{2} \bar{f}_{\alpha}^{(\mu)} f_{\alpha}^{(\mu)} a_{\alpha}^{\dagger} a_{\alpha}-C_{\alpha}^{(\mu)} a_{\alpha}^{\dagger} a_{\alpha}\right), \\
& X_{\alpha}^{(\mu)}=x^{\mu} a_{\alpha}^{\dagger} a_{\alpha}
\end{aligned}
$$

for $\mu=0,1, \ldots, n-1$. Here, $W_{\alpha}^{(\mu)}$ and $C_{\alpha}^{(\mu)}$ are constant functions with respect to $x^{\mu}$ and may depend arbitrarily on the parameters $x^{\nu}$ with $\nu \neq \mu$. The operators $\mathcal{F}_{\alpha}^{(\mu)}$ are hemitean only if both of $W_{\alpha}^{(\mu)}$ and $C_{\alpha}^{(\mu)}$ are real. There is an orthonormal and complete set of states which are eigenstates of the operators $\mathcal{F}_{\alpha}^{(\mu)}, N_{\alpha}$ and $X_{\alpha}^{(\mu)}$ since

$$
\left[X_{\alpha}^{(\mu)}, X_{\beta}^{(\nu)}\right]=\left[\mathcal{F}_{\alpha}^{(\mu)}, \mathcal{F}_{\beta}^{(\nu)}\right]=\left[X_{\alpha}^{(\mu)}, N_{\alpha}\right]=\left[\mathcal{F}_{\alpha}^{(\mu)}, X_{\beta}^{(\nu)}\right]=\left[\mathcal{F}_{\alpha}^{(\mu)}, N_{\alpha}\right]=0
$$

as a consequence of the independence of the modes.

If the operator $\partial X_{\alpha}^{\mu}$ is invertible it is possible to obtain the equation of motion for $\mathcal{F}_{\alpha}^{(\mu)}$ in the space of modes. Like in the classical case we first determine $\partial X_{\alpha}^{\mu}$ and $\partial^{2} X_{\alpha}^{\mu}$ and we obtain the following relations

$$
\partial X_{\alpha}^{\mu}=-\frac{2}{W_{\alpha}^{(\mu)}}\left[\left(\frac{1}{2}+\frac{C_{\alpha}^{(\mu)}}{\bar{f}_{\alpha}^{(\mu)} f_{\alpha}^{(\mu)}}\right) \partial^{2} \mathcal{F}_{\alpha}^{(\mu)} \phi_{\alpha}^{(\mu)}+\left(\frac{1}{2}-\frac{C_{\alpha}^{(\mu)}}{\bar{f}_{\alpha}^{(\mu)} f_{\alpha}^{(\mu)}}\right) \partial^{2} \mathcal{F}_{\alpha}^{(\mu)} \phi_{\alpha}^{(\mu)}\right]
$$

and

$$
\partial^{2} X_{\alpha}^{\mu}=-\frac{2}{W_{\alpha}^{(\mu)}}\left(\frac{1}{2}+\frac{C_{\alpha}^{(\mu)}}{\bar{f}_{\alpha}^{(\mu)} f_{\alpha}^{(\mu)}}\right) \partial^{3} \mathcal{F}_{\alpha}^{(\mu)} \phi_{\alpha}^{(\mu)}-\frac{4}{W^{(\mu)}}\left(\frac{C_{\alpha}^{(\mu)}}{\bar{f}_{\alpha}^{(\mu)} f_{\alpha}^{(\mu)}}\right) \partial^{2} \mathcal{F}_{\alpha}^{(\mu)} .
$$

Next introduce (24) and (25) in the operatorial equation corresponding to (2) and express the derivatives $\partial_{\mu}$ in terms of $\partial / \partial \phi_{\alpha}^{(\mu)}$. For the sake of clarity let us introduce the following notations for the functions that enter the final result

$$
\begin{aligned}
\Sigma & =\left(\bar{f}_{\alpha}^{(\mu)} f_{\alpha}^{(\mu)}\right)^{-1}, \quad \Omega_{1}=-\frac{2}{W_{\alpha}^{(\mu)}}\left(\frac{1}{2}+\frac{C_{\alpha}^{(\mu)}}{\bar{f}_{\alpha}^{(\mu)} f_{\alpha}^{(\mu)}}\right) \\
\Omega_{2} & =-\frac{4}{W^{(\mu)}}\left(\frac{C_{\alpha}^{(\mu)}}{\bar{f}_{\alpha}^{(\mu)} f_{\alpha}^{(\mu)}}\right) .
\end{aligned}
$$

Next we drop the indices $\mu$ and $\alpha$ and put a hat on operators. With these notations the equation satisfied by the quantum prepotentials has the following form

$$
\begin{aligned}
M \Sigma \partial \hat{\mathcal{F}} \hat{\phi} \hat{Y}\left[\left(\left(\Sigma \left(\partial^{2} \hat{\mathcal{F}} \hat{\phi}\right.\right.\right.\right. & \left.\left.+\partial \hat{\mathcal{F}})-\Sigma \partial \hat{\mathcal{F}} \hat{\phi} \hat{Y}\left(\Omega_{1} \partial^{3} \hat{\mathcal{F}} \hat{\phi}+\Omega_{3} \partial^{2} \hat{\mathcal{F}}\right)\right) \hat{Y}+1\right) \partial^{2} \hat{\mathcal{F}} \\
& \left.+\Sigma \partial \hat{\mathcal{F}} \hat{\phi} \hat{Y} \partial^{3} \hat{\mathcal{F}}\right]+P \partial \hat{\mathcal{F}}+Q=0
\end{aligned}
$$


where $\hat{Y}=(\partial \hat{X})^{-1}$. Equation (27) is nonlinear and nontrivial, however some simplifications are possible in the homogeneous case and by taking the constant $C_{\alpha}^{(\mu)}=0$. Nevertheless, even in the simplest case it is a very difficult task to find a nontrivial solution of it.

The equation (22) and (27) represent the content of the duality between the coordinates and fields in the quantum theory. This duality was obtained by imposing strong constraints on the system. The reason for these constraints is the requirement that the duality has the same interpretation as in the classical theory in the Minkowski spacetime [1] (see also [16].)

In conclusion, we have generalized the classical duality between coordinates and matter fields to curved spacetime. In the classical case, in order to obtain a theory that reduces to the one discussed in [1], the constraints expressed by (6) and (12) should be imposed on the system. Moreover, in order to apply the method used to derive the flat spacetime duality, one should assume that the system satisfies some restrictive mathematical conditions. In the quantum case, the duality exists on those manifolds on which the local decomposition of the scalar fields in independent modes is possible along each coordinate. We obtained in particular the relations derived in the case of Minkowski spacetime in [1]. Our analysis shows that the duality coordinate-fields should also hold locally in the case of other flat manifolds. However, we emphase that this is not a property of general spacetime manifolds, at least not in the form of [1]. We have shown that, in principle, this type of duality should hold for some nontrivial manifolds but the complexity of the matematical restrictions that should be imposed to the system prevents us of giving examples at present.

Despite the technical difficulties, the theory presented here might be useful in studying the general relativity at Planck scale. One interesting way to apply the coordinate-field duality to gravity would be to parametrize locally the spacetime manifold in terms of matter fields in a consistent way with the principles of general relativity. 


\section{ACKNOWLEDGMENTS}

We thank to S. Sorella and J. A. Helayel-Neto for important discussions. M. A. A. is

grateful to CBPF for support. I. V. V. thanks to CBPF and UCP for hospitality. I. V. V.'s work was partially supported by a FAPERJ and a FAPESP postdoc fellowships. 


\section{REFERENCES}

[1] A. E. Faraggi and M. Matone, Phys. Rev. Lett. 78, 163 (1997).

[2] N. Seiberg and E. Witten, Nucl. Phys. B 426, 19 (1994).

[3] G. Bonelli and M. Matone, Phys. Rev. Lett. 76, 4107 (1996).

[4] M. Matone, Phys. Lett. B 357, 342 (1995).

[5] S. Doplicher, K. Fredenhagen and J. E. Roberts, Phys. Lett. B 331, 39 (1994); Comm. Math. Phys. 172, 187 (1995).

[6] J. Madore, Ann. Phys. 219, 187 (1992).

[7] A. H. Chamseddine and A. Connes, Phys. Rev. Lett. 74, 4868, (1996); Comm. Math. Phys. 186, 731 (1997).

[8] A. Connes, Noncommutative Geometry (Academic Press, 1994); G. Landi, An Introduction to Noncommutative Spaces and their Geometries (Springer-Verlag, 1997).

[9] R. M. Wald, Quantum Field Theory in Curved Spacetime and Black Holes(University of Chicago Press, 1994); N. D. Birrell and P. C. W. Davies, Quantum Fields in Curved Space(Cambridege, 1989).

[10] G. Landi and C. Rovelli, Phys. Rev. Lett. 78, 3051 (1997); Mod. Phys. Lett. A 13, 479 (1998)

[11] G. Landi, gr-qc/9906044

[12] I. V. Vancea, Phys. Rev. Lett. 79, 3121 (1997), Errattum-ibid80, 1355 (1998); Phys. Rev. D 58, 045005 (1998)

[13] C. Ciuhu and I. V. Vancea, gr-qc/9807011.

[14] N. Pauna and I. V. Vancea, Mod. Phys. Lett. A 13, 3091 (1998). 
[15] W. Lederman and S. Vajda, Handbook of Aplicable Mathematics, Vol. IV, (John Wiley and Sons, 1982).

[16] M. A. De Andrade and I. V. Vancea, Coordinate-Field Duality and Quantum Field Theory, in preparation. 